\title{
Numerical tools for the theoretical study of QCD at small $x$
}

\author{
A. Sabio Vera \\ Physics Department, Theory Division, CERN, CH-1211 Geneva 23, Switzerland \\ E-mail: Agustin.Sabio.Vera@cern.ch \\ P. Stephens ${ }^{* \dagger}$ \\ Institute of Nuclear Physics, Polish Academy of Sciences, \\ ul. Radzikowskiego 152, 31-342 Cracow, Poland \\ E-mail: pstephens@annapurna.ifj.edu.pl
}

\begin{abstract}
In this contribution we present the status of two numerical tools designed to study the small $x$ limit of QCD. The first one is a Monte Carlo simulation of the BFKL evolution equation. In design of this approach emphasis has been placed on exploiting the linear behaviour that many variants of the BFKL evolution possess. This allows us to design a procedure which can be used to study theoretical and phenomenological aspects of different kernels. The second one is a semi-analytic approach to study Lipatov's effective action which describes Reggeon interactions. The study of the properties of this action is very complicated and we propose using a computational tool to handle the large amount of non-local vertices and the derivation of higher order corrections.
\end{abstract}

DIFFRACTION 2006 - International Workshop on Diffraction in High-Energy Physics

September 5-10 2006

Adamantas, Milos island, Greece

\footnotetext{
* Speaker.

${ }^{\dagger}$ This work is partly supported by the EU grant mTkd-CT-2004-510126 in partnership with the CERN Physics Department and by the Polish Ministry of Scientific Research and Information Technology grant No 620/E-77/6.PRUE/DIE 188/2005-2008.
} 


\section{Introduction}

The structure of Quantum Chromodynamics is very rich. Besides the intrinsic uncertainties for the description of the theory in the nonperturbative region, the regime where the coupling is small also presents many challenges. In order to describe some processes dominated by perturbative dynamics it has been important to focus the efforts on different regions of phase space where logarithmic factors get enhanced and their resummation to all orders can provide valuable information. One example of this kind is the Regge limit of strong interacting processes. This is a regime where the center of mass energy, $s$, is very large and terms of the form $\alpha_{s} \ln s$ are enhanced. In processes characterized by two large and similar transverse scales, for a range of large energies, the underlying dynamics is linear. This implies that the growth with energy of the observables can be described by a linear integral equation, the so-called Balitsky-Fadin-Kuraev-Lipatov (BFKL) equation [1]. Linearity has, as a consequence, an exponential rise of the cross section with an exponent related to the so-called hard pomeron intercept. The LO intercept is too large when compared to experimental data. The inclusion of the next-to-leading (NLO) order corrections [2], where there is an extra power in the coupling when compared to the logs of energy, reduces the intercept to values more acceptable from the point of view of phenomenology. The BFKL scattering amplitudes contain a large amount of physical information which can be fully extracted only by numerical methods. It has a Poisson like structure in the number of emissions which could be useful in the study of events with a large multiplicity in the final state. At NLO one is sensitive to effects like the running of the coupling or how to best couple the Reggeized gluons to the external particles. These issues can be best treated in a numerical way using the methods developed in Ref. [3]. Here we will review some of the equations shown in these works and we will introduce a more efficient implementation, for alternative ideas see Ref. [4]. The use of the tools shown here is not limited to the standard forward BFKL kernel but it can be extended to the nonforward case which corresponds to colour singlet exchange, and more exotic situations as is the case when the QCD evolution is placed in a thermal bath.

Although there should be a window of large energies where BFKL effects are dominant, linearity leads to the breakdown of unitarity since the Froissart bound is not respected for asymptotic energies. This is connected to the well-known sentence by the late economist Kenneth E. Boulding: "Anyone who believes exponential growth can go on forever in a finite world is either a madman or an economist". Therefore at some point unitarity corrections should be taken into account. This is a very complicated issue from the theoretical point of view and all proposed solutions are always approximations. We believe an attractive approach is that proposed by L. Lipatov in Ref. [5]. A non-linear gauge invariant effective action was constructed which describes the interactions between Reggeized quarks and gluons with the usual ones. The Reggeized degrees of freedom are represented by Wilson lines while the $s$-channel emissions belong to a kinematics more general than the quasi-multi-Regge region needed to obtain the NLO kernel in the sense that not only a single pair of partons is allowed to have a fixed invariant mass but more are allowed. These "grouped" emissions are well separated from each other in rapidity following the usual multi-Regge kinematics. This action incorporates unitarity in different channels and describes the interaction between Reggeons as well as, through quantum fluctuations around the classical solution, higher order corrections to the BFKL kernel. In this paper we propose the use of a second tool which uses the 
semi-analytic software Effective [12] to study and probe the tree-level structure of this effective action. In combination with a topology generator, the effective vertices of Reggeons coupled to particles can be generated automatically.

\section{Monte Carlo implementation of BFKL equations}

The first work taking full advantage of the iterative structure present in the BFKL equation to develop a Monte Carlo solution for the gluon Green's function was presented in Ref. [6]. More recently this method was extended to the much more complicated case of the NLO kernel in Ref. [3]. Here we would like to report on recent progress on a new implementation of these ideas to continue investigating the gluon Green's function in different physical processes.

The LO evolution equation can be written in a very simple form as

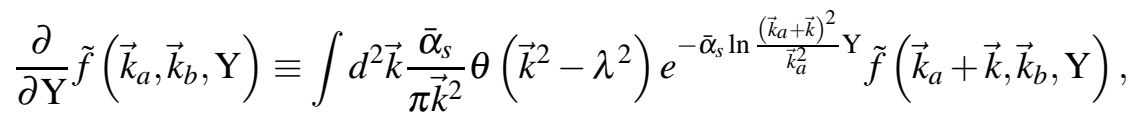

if the inital condition

$$
\tilde{f}\left(\vec{k}_{a}, \vec{k}_{b}, \mathrm{Y}\right)=\delta^{(2)}\left(\vec{k}_{a}-\vec{k}_{b}\right),
$$

is chosen for a function related to the gluon Green's function up to a factor including the Reggeized gluon propagator, i.e.

$$
f\left(\vec{k}_{a}, \vec{k}_{b}, \mathrm{Y}\right) \equiv e^{\omega_{0}\left(\vec{k}_{a}^{2}, \lambda^{2}\right) \mathrm{Y}} \tilde{f}\left(\vec{k}_{a}, \vec{k}_{b}, \mathrm{Y}\right) .
$$

The generalization of this formula to the case of colour singlet exchange is straightforward [7] and the evolution equation can be written as

$$
\begin{aligned}
& \frac{\partial}{\partial \mathrm{Y}} \tilde{f}\left(\vec{k}_{a}, \vec{k}_{b}, \vec{q}, \mathrm{Y}\right) \equiv \int d^{2} \vec{k} \frac{\bar{\alpha}_{s}}{2 \pi \vec{k}^{2}}\left(1+\frac{\vec{k}_{a}^{* 2}\left(\vec{k}_{a}+\vec{k}\right)^{2}-\vec{q}^{2} \vec{k}^{2}}{\vec{k}_{a}^{2}\left(\vec{k}_{a}^{*}+\vec{k}\right)^{2}}\right) \theta\left(\vec{k}^{2}-\lambda^{2}\right)
\end{aligned}
$$

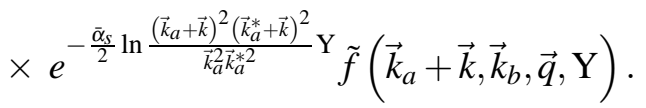

The generalization for the NLO case has a very similar structure although the kernels and gluon Regge trajectories are more complicated.

The numerical results are presented in fig. 1. These correspond to the LO forward case with $Y=4$ and $\vec{k}_{a}=(50,0) \mathrm{GeV}$. Work is in progress to implement other cases at LO and NLO as well. In these figures the analytic solution is found from the sum of conformal spins up to 20 and the maximum number of emissions in the Monte Carlo implementation is 30 . As can be seen there is still a small discrepancy as the ratio $k_{b}^{2} / k_{a}^{2}$ moves away from 1 . This is still under investigation.

One of the questions we would like to answer is what is the effect of the collinear resummation in $k_{t}$-space proposed in Ref. [8] on the behaviour of the Green's function when the value of the transverse momenta in the incoming Reggeons is very different. It is well-known that for larger values of the coupling this region presents an instability in terms of convergence and it would be important to understand how to solve this situation in a fully exclusive way by complementing the 

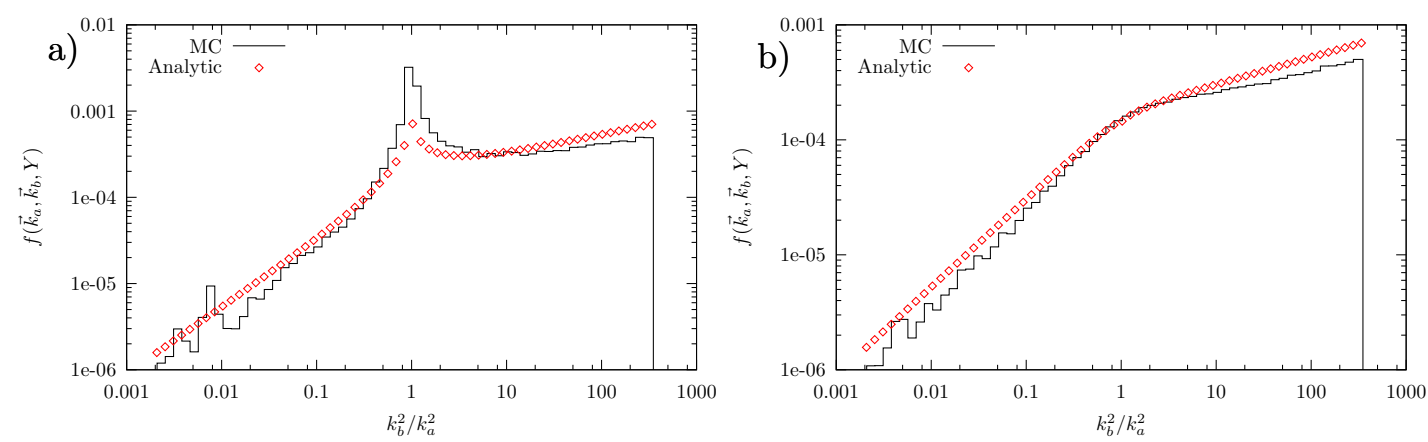

Figure 1: Comparison of LO forward analytic versus MC for a fixed angles a) $\phi=0$ b) $\phi=\pi / 4$. For these figures we take $Y=4$ and $\vec{k}_{a}=(50,0) \mathrm{GeV}$. The source of the discrepancy, in both figures, away from $k_{b}^{2} / k_{a}^{2}=1$ is still under investigation, as is the ehancement for $\phi=0$ around $k_{b}^{2} / k_{a}^{2}=1$.

BFKL approach with all-order contributions dominant in the collinear region. The prescription proposed in [8] has a simple implementation since it suggests that the modification needed in the NLO kernel to introduce the collinear improvements is to remove the term

$$
-\frac{\bar{\alpha}_{s}^{2}}{4} \frac{1}{\pi(\vec{q}-\vec{p})^{2}} \ln ^{2}\left(\frac{q^{2}}{p^{2}}\right)
$$

in the NLO real emission kernel and replace it with

$$
\begin{gathered}
\frac{1}{\pi(\vec{q}-\vec{p})^{2}}\left\{\left(\frac{q^{2}}{p^{2}}\right)^{-\mathrm{b} \bar{\alpha}_{s} \frac{|p-q|}{p-q}} \sqrt{\frac{2\left(\bar{\alpha}_{s}+\mathrm{a} \bar{\alpha}_{s}^{2}\right)}{\ln ^{2}\left(\frac{q^{2}}{p^{2}}\right)}} J_{1}\left(\sqrt{2\left(\bar{\alpha}_{s}+\mathrm{a} \bar{\alpha}_{s}^{2}\right) \ln ^{2}\left(\frac{q^{2}}{p^{2}}\right)}\right)\right. \\
\left.-\bar{\alpha}_{s}-\mathrm{a} \bar{\alpha}_{s}^{2}+\mathrm{b} \bar{\alpha}_{s}^{2} \frac{|p-q|}{p-q} \ln \left(\frac{q^{2}}{p^{2}}\right)\right\}
\end{gathered}
$$

where

$$
\mathrm{a}=\frac{5}{12} \frac{\beta_{0}}{N_{c}}-\frac{13}{36} \frac{n_{f}}{N_{c}^{3}}-\frac{55}{36}, \mathrm{~b}=-\frac{1}{8} \frac{\beta_{0}}{N_{c}}-\frac{n_{f}}{6 N_{c}^{3}}-\frac{11}{12}
$$

are the collinear coefficients of the kernel in Mellin space.

Recently a theoretical derivation of a jet definition valid at NLO has been calculated in the context of the NLO BFKL equation in Ref. [10]. One of the targets of our research will be to implement this jet definition and study jet multiplicities of fully generated events at NLO. Issues related to the conservation of longitudinal components in the final state emissions can be studied in this context.

\section{Reggeon Effective Action}

The Reggeon effective action [11] contains a large quantity of physics. The problem is how to retrieve the interesting and possibly new physics from this action. This is still a new area of work and there is little experience about the best path to proceed down. We have decided to develop a computational tool to help us understand this action and study its behaviour. 
The action is the standard QCD action plus a term induced by the reggeization of the gluon. This term can be expressed in terms of a kinetic term, describing the propagation of a Reggeon field, and Reggeon-gluon coupling terms

$$
\mathscr{L}_{\text {ind }}=\mathscr{L}_{\text {ind }}^{k}+\mathscr{L}_{\text {ind }}^{R G}
$$

In order to discuss these terms, we must introduce the kinematics implicit in this action. The action is defined in the quasi-multi-Reggeon kinematics (QMRK) approximation. In this regime the final states are produced in clusters, with each cluster having total momentum $Q_{i}$. Cluster $k$ is composed of any number of gluons, each containing momentum $p_{j}^{(k)}$. Therefore we have

$$
\begin{aligned}
P_{A}+P_{B} & =Q_{1}+Q_{2}+\ldots+Q_{n} ; Q_{i}^{2}=M_{i}^{2}, Q_{k}=\sum_{j} p_{j}^{(k)}, \\
s & =2 P_{A} P_{B}=4 E^{2} \gg s_{i}=2 Q_{i} Q_{i+1} \gg\left|t_{i}\right|=\left|q_{i}^{2}\right|,
\end{aligned}
$$

where $P_{A}$ and $P_{B}$ are incoming momenta. We now introduce the light-cone vector

$$
n_{ \pm}^{\mu}=P_{B / A}^{\mu} / E ;\left(n^{ \pm}\right)^{2}=0 ; n^{+} \cdot n^{-}=2 ; n^{ \pm} \cdot \vec{k}_{T}=0 .
$$

We can now use this projection to project the Lorentz derivatives and define the inverse projected Lorentz derivative acting on a field

$$
\partial_{ \pm}=n_{ \pm}^{\mu} \partial_{\mu} ; \frac{1}{\partial_{ \pm}} A^{\mu}=\frac{i}{p_{ \pm}} A^{\mu}
$$

Using these definitions the kinetic term is

$$
\mathscr{L}_{\text {ind }}^{k}=-\partial_{+} R^{-} \partial_{-} R^{+}
$$

for Reggeon field $R$. In the QMRK the Reggeon momentum is transverse. This is given by the condition $\partial_{ \pm} R^{ \pm}=0$ which is inherently included in eqn. (3.6). The induced couplings between Reggeon fields and gluonic fields is then

$$
\mathscr{L}_{\text {ind }}^{R G}=-\operatorname{Tr}\left\{\frac{\partial_{+}}{g}\left[\mathscr{P} \exp \left(-\frac{1}{2} \int_{-\infty}^{x^{+}} A_{+}\left(x^{\prime}\right) d x^{\prime+}\right)\right] \partial^{2} R_{-}(x)+(-\leftrightarrow+)\right\} .
$$

For notational simplicity we have defined

$$
A_{\mu}=-i T^{a} A_{\mu}^{a}, R_{ \pm}=-i T^{a} R_{ \pm}^{a}
$$

where $T^{a}$ are the $S U(3)$ generators in the fundamental representation. The trace is over colour indices. This path exponentiated integral can be written in the form

$$
\frac{\partial_{+}}{g} \mathscr{P} \exp \left(-\frac{1}{2} \int_{-\infty}^{x^{+}} A_{+}\left(x^{\prime}\right) d x^{\prime+}\right)=A_{+} \sum_{i=0}^{\infty}\left(\frac{-g}{\partial_{+}} A_{+}\right)^{i},
$$

and can then be used to define the interaction vertices of the Reggeon field with the gluonic fields.

We wish to be able to use the action to calculate some observables. In order to do this a convenient tool to have is the effective vertices which couple $n$ Reggeon fields to $m$ gluonic fields. 


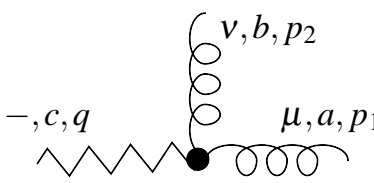

(T1)

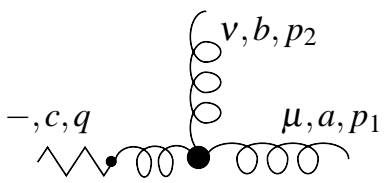

(T2)

Figure 2: Topology of a minus Reggeon to two gluons. Zig-zag line is a Reggeon while swirly lines are gluons.

This requires generating the correct topologies and then extracting from eqn. (3.7) the bare vertices and applying them to the topology. For this task we are using the analytic effective action analysis tool Effective [12]. This tool allows us to define the Lagrangian (with the additional definitions of the operators) and extract the appropriate couplings from this action. We couple this to a topology generator to produce the tree-level effective vertices for $n$ Reggeon and $m$ gluons.

We will give now an example of calculating the effective vertex which couples a Reggeon with minus light-cone component to two gluons. Figure 2 shows the topologies of this effective action. We see that this has two contributions. The first is the bare vertex which can be found in the action. We see, in momentum space, this is simply

$$
\mathscr{L}_{\text {ind }}^{R G G}=-i q^{2} g\left(\frac{A_{+}\left(p_{1}\right) A_{+}\left(p_{2}\right) R_{-}(q)}{p_{i}^{+}}+\frac{A_{-}\left(p_{1}\right) A_{-}\left(p_{2}\right) R^{+}(q)}{p_{i}^{-}}\right),
$$

where $i$ can be the momentum of either gluon. The colour factor must also be extracted from these terms. Doing so we find

$$
A_{+}\left(p_{1}\right) A_{+}\left(p_{2}\right) R_{-}(q)=(-i)^{3} T^{a} T^{b} T^{c} A_{a}^{+}\left(p_{1}\right) A_{b}^{+}\left(p_{2}\right) R_{c}^{-}(q) .
$$

Using the condition $p_{1}^{-}+p_{2}^{-}=0$ for the minus Reggeon and

$$
A^{\mu}=\frac{1}{2}\left(n^{-}\right)^{\mu} A^{+}+\frac{1}{2}\left(n^{+}\right)^{\mu} A^{-}+A^{T},
$$

we can sum the two contributions (choice of $i$ in momentum) to find

$$
\left\langle 0\left|\mathscr{L}_{\text {ind }}^{R G}\right| A_{a}^{\mu}\left(p_{1}\right) A_{b}^{v}\left(p_{2}\right) R_{c}^{-}(q)\right\rangle=i g q^{2} f_{a b c}\left(n^{-}\right)^{\mu}\left(n^{-}\right)^{v} \frac{1}{p_{1}^{-}} .
$$

For the other topology we have a Reggeon-gluon transition which can be found from the $\mathscr{O}\left(g^{0}\right)$ term in the induced action, and a standard QCD three-gluon vertex. The transition term simply provides a coupling $-q^{2} \frac{\delta^{a a^{\prime}}}{2}\left(n^{-}\right)^{\lambda}$. The three-gluon coupling vertex is

$$
g f_{a b c}\left[\left(q-p_{1}\right)_{v} g_{\lambda \mu}+\left(p_{1}-p_{2}\right)_{\lambda} g_{\mu \nu}+\left(p_{2}-q\right)_{\mu} g_{v \lambda}\right] .
$$

Using these we find the contribution from the (T2) topology

$$
g f_{a b c}\left[\left(p_{1}-p_{2}\right)^{-} g^{\mu v}+\left(-2 p_{1}-p_{2}\right)^{v}\left(n^{-}\right)^{\mu}+\left(2 p_{2}+p_{1}\right)^{\mu}\left(n^{-}\right)^{v}\right],
$$

which can be combined with eqn. (3.13) to give the full effective vertex

$$
V_{a b c}^{\mu v-}=g f_{a b c}\left[\left(p_{1}-p_{2}\right)^{-} g^{\mu v}+\left(-2 p_{1}-p_{2}\right)^{v}\left(n^{-}\right)^{\mu}+\left(2 p_{2}+p_{1}\right)^{\mu}\left(n^{-}\right)^{v}-\frac{q^{2}}{p_{1}^{-}}\left(n^{-}\right)^{\mu}\left(n^{-}\right)^{v}\right] .
$$


The software package being implemented will calculate the effective vertices in the same manner as what is presented here. We plan to use our code to verify the other vertices found in [11], rather than calculating them by hand. We also plan to calculate new vertices which have not been presented in [11]. Using an automated system has the additional advantage that it may allow us to integrate with other codes, e.g. matrix element generators.

\section{Outlook}

We have presented the preliminary work on two computational tools. The first is a Monte Carlo to compute the solution to the BFKL equation for several different kernels. We also want this code to be able to produce fully exclusive events at NLO, but this requires still a lot of work. We have shown the results of our current implementation for the LO forward kernel and discussed the other kernels which are of interest to us.

The second part of these proceedings introduces another computational tool designed to begin the analysis of the effective action proposed in [11]. This action is quite complicated, but may include important and interesting physics. Because of the complexity to analyze the action, we feel a computational tool is ideally suited for this purpose. Thus we introduce the code which we are currently writing to analyze this action. We also present an example of the type of calculation which this code will perform.

Acknowledgments: Discussions with J. Bartels and L. Lipatov are acknowledged. PS would also like to thank the conference organizers for their invitation and financial support.

\section{References}

[1] L. N. Lipatov, Sov. J. Nucl. Phys. 23, 338 (1976); V. S. Fadin, E. A. Kuraev and L. N. Lipatov, Phys. Lett. B 60, 50 (1975), Sov. Phys. JETP 44, 443 (1976), Sov. Phys. JETP 45, 199 (1977); I. I. Balitsky and L. N. Lipatov, Sov. J. Nucl. Phys. 28, 822 (1978), JETP Lett. 30, 355 (1979).

[2] V.S. Fadin, L.N. Lipatov, Phys. Lett. B 429, 127 (1998); G. Camici, M. Ciafaloni, Phys. Lett. B 430, 349 (1998).

[3] J. R. Andersen, A. Sabio Vera, Phys. Lett. B 567, 116 (2003); Nucl. Phys. B 679, 345 (2004); Nucl. Phys. B 699, 90 (2004); JHEP 0501, 045 (2005).

[4] J. R. Andersen, Phys. Lett. B 639, 290 (2006).

[5] L. N. Lipatov, Nucl. Phys. B 452, 369 (1995).

[6] C. R. Schmidt, Phys. Rev. Lett. 78, 4531 (1997).

[7] J. R. Andersen and A. Sabio Vera, JHEP 0501, 045 (2005).

[8] A. Sabio Vera, Nucl. Phys. B 722, 65 (2005).

[9] A. Sabio Vera, Nucl. Phys. B 746, 1 (2006).

[10] J. Bartels, A. Sabio Vera and F. Schwennsen, hep-ph/0608154.

[11] E. N. Antonov, L. N. Lipatov, E. A. Kuraev and I. O. Cherednikov, Nucl. Phys. B 721, 111 (2005) [arXiv:hep-ph/0411185].

[12] J. P. J. Hetherington and P. Stephens, arXiv:hep-ph/0605149. 